\title{
Introducing particle interphase model for describing the electrical behaviour of nanodielectrics
}

\author{
Fuad N. Alhabill*1, 2, Raed Ayoob ${ }^{1}$, Thomas Andritsch' ${ }^{1}$, and Alun S. Vaughan ${ }^{1}$ \\ ${ }^{1}$ Tony Davies High Voltage Laboratory, Electrical Power Engineering Group, University of \\ Southampton, Southampton, SO17 1BJ, United Kingdom \\ ${ }^{2}$ Department of Engineering and Design, University of Chichester, Chichester, United Kingdom \\ Corresponding author: E-mail: f.alhabill@chi.ac.uk (F. N. Alhabill)
}

\begin{abstract}
This study proposes a new model for describing the electrical behavior of nanocomposites. Unlike other models in the literature, this model has concentrated on the role of an interphase layer within the boundaries of nanoparticles. The experimental part investigates this role by filling an epoxy matrix with two types of surface-modified silicon nitride nanofiller: (a) the particles were dried at $200{ }^{\circ} \mathrm{C}$, and (b) the particles were calcinated at $1050{ }^{\circ} \mathrm{C}$. Electrical characterization showed that the epoxy which was filled with the calcinated particles has considerably better dielectric performance. Given that thermal and dielectric spectroscopy results demonstrate that the matrix molecular dynamics and polar content are comparable for all the investigated samples, the variations in the dielectric performance point to the particle interphase as an essential reason. As shown by infrared spectroscopy, the complex surface chemistry of the dried particles suggests a particle interphase with a high concentration of localized electronic states, which may enhance charge transport through hopping/tunnelling conduction. On the other hand, calcinating the particles results in a particle interphase with wider band gap, which may work as an energy barrier for charge movement. Consequently, this study highlights the paramount importance of particle interphase for designing dielectric properties of nanodielectrics.
\end{abstract}

KEYWORDS: Nanodielectrics, surface chemistry, interface, polymer interphase, particle interphase, dielectric properties.

\section{Introduction}

The reliable production of electrical energy without causing adverse environmental consequences is of major global concern, since energy consumption is generally increasing and areas such as transport, which were previously the domain of fossil fuels, become increasingly electrified. This necessitates increased electric power generation, transmission and distribution capacities which, consequently, necessitates improved insulation materials. The commercial impact of even incremental improvement in dielectric properties of the insulation materials is potentially very large since the quantity of electrical insulation in use is vast. The potentials of introducing nanoparticles into a dielectric material for electrical insulation applications were theoretically highlighted by Lewis in 1994 [1]. However, nanodielectrics did not draw a lot of attention until 2002 when Nelson et al. [2] had experimentally demonstrated some advantages for nanofilled materials over microfilled counterparts, such as reducing space charge accumulation. Since 
then, a burgeoning interest in the subject has been shown trying to explore the potentials of nanodielectrics and investigate the underlying mechanisms that control the behaviour of these systems. Many of these factors have been empirically identified over the last two decades; however, detailed information about the underlying physics and chemistry behind these factors needs more research. These factors include the following:

- Particle dispersion: considering their large specific surface area, the friction and surface tension forces between nanoparticles are dramatically higher than between microparticles, for example. This makes the nanoparticles stick together and complicates their dispersion [3]. Nanoparticle agglomeration may result in a large enough particle to disturb and locally enhance the electric field due to the differences in the conductivity and permittivity between the filler and matrix materials, an analog to what occurs in microcomposite systems [4-6].

- Local, interfacial interactions and interphase formation: the interactions between the particles and the surrounding matrix result in forming such region. The characteristics and influence of the interphase regions will be discussed in detail in the following paragraph.

- Changes in matrix structure: introducing nanoparticles into a polymeric matrix might alter its morphological structure, particularly for semicrystalline systems like polyethylene [4, 7-9]. Hosier [10] experimentally demonstrated that the breakdown strength can be affected by modifying the spherulitic morphology of the unfilled polyethylene. Therefore, the changes in the dielectric performance of the nanocomposites based on semicrystalline polymers might be greatly affected by perturbations to the crystalline morphology, rather than being associated with the particles themselves.

- Environmental factors, notably, absorption of water: many of the widely utilized nanoparticles, due to their surface chemistry, have the tendency to adsorb water molecules on their surface, which results in a water layer surrounding the nanoparticles. These water layers have a detrimental impact on the dielectric performance of the nanocomposites as reported [11-14].

All the above factors should be kept in mind when trying to design/analyze the effect of introducing nanoparticles into a polymeric matrix, as some of these factors may dominate others under particular circumstances. That is, it is unlikely that any single factor is likely to provide a ubiquitous explanation across all material systems for all properties. For example, the negative dielectric consequences caused by particles' water absorption might dwarf any dielectric improvement caused by the introduction of the nanoparticles [15]. The relevance or the weight of each factor are functions of the matrix material, filler characteristics and the interactions between them.

The key feature that differentiates nanocomposites from microcomposites is the vastly greater specific interfacial area between the particles and the encapsulating polymer [16, 17]. The layers that are adjacent to the interface might be affected by being next to different phase and to the interactions that occur between the two phases. These interactions are proposed to result in the formation of an interphase region with modified properties [18-20]. Consequently, as the size of the particles becomes smaller, the specific surface interfacial area will increase and, consequently, any interphase regions will constitute a commensurately larger volume fraction of the whole system which, it has been argued, explains how the macroscopic properties of nanocomposite differ from those of either component in isolation. Different models have been suggested to highlight the main characteristics of the interphase region and to describe the interactions that may occur in this region. However most of these models, i.e. Lewis intensity model $[21,22]$ and electric double layer model $[22,23]$, do not offer a critical understanding of the interphase region, instead, they just qualitatively describe it without distinct experimental or analytical evidence. The 
multicore model [24] presents a more explicit mechanism for describing the potential influence of the nanoparticles on the molecular dynamics of the polymeric matrix, particularly when the particles are bonded to the polymer. Many experimental studies have investigated the effect of the nanofiller on the polymeric matrix by observing the molecular dynamics over the glass transition process using techniques such as differential scanning calorimetry or dielectric spectroscopy. Some of these studies did not detect any evidence for such effects, even where attractive interactions between the filler and the polymer, i.e. strong hydrogen bonding, are present $[25,26]$. Therefore, the multicore model cannot be used to fully describe these nanocomposites. Other studies do detect an immobilized layer [27, 28], that does not contribute to the glass transition of the rest of the matrix, or a layer with different molecular dynamics around the particles [29, 30]. However these effects were marked at a high loading ratio, > $20 \mathrm{wt}$ \% for nanofiller less than $15 \mathrm{~nm}$ in diameter, and hence, the thickness of the confined layer was estimated to be less than $\sim 2 \mathrm{~nm}[27,28]$, much less than that anticipated by the multicore model. More importantly, direct linkage between molecular dynamics effects and the dielectric performance is neither clear nor experimentally proven. For example, changing the segmental dynamics by changing the crosslinking density in an epoxy matrix did not correlate with the dielectric performance [31]. Even for nanocomposites where the filler has an impact on the cooperative relaxation at the glass transition process, the $\beta$ relaxation, which is associated with smaller segmental dynamics, was not perturbed [27, 28]. For this relationship, the multicore model proposed that an electric double layer is superimposed on the multicore model. Additionally, the authors speculated that the strain on the confined polymeric segments around the nanoparticles affects the free volume content. No experimental evidence supports these claims. On the contrary, a recent study [32] has demonstrated that the free volume is little affected by the addition of nanoparticles, and cannot account for the significant variations seen in the dielectric strength for many nanodielectrics. As such, a mature understanding of the interphase region characteristics is not yet achieved. A detailed model that can relate the macroscopic behaviour of nanodielectrics with the interactions between the particles and the encapsulating polymer is needed in order to open the potential of these composites to be tailored and designed, and to meet our future demands on dielectrics. For underlying the dielectric behaviour, which is concerned with the dynamics of as small species as electrons, the prospective model might need to investigate the effect of the interactions at the microscopic level [16]. For example, these interactions might affect the density of states in the interphase region [16], which consequently influences the overall electronic mobility in the system.

Another element that can be emphasized about current nanodielectrics models is that, until now, these models have concentrated on the interphase region inside the polymer, the "polymer interphase" in Figure 1 , neglecting any interphase layer inside the boundaries of the particles themselves, "particles interphase" in Figure 1. This might be because the molecules of the filler materials, usually ceramic materials, are very small compared with the polymer molecules or chains, thus, the particle interphase is expected to be much thinner than the polymer interphase. Nevertheless, the higher density of the particle materials implies denser electronic energy states and, consequently, any perturbations in the electronic states in the particles interphase, such as changing their energy levels or spatial extension, might have a more profound impact on the dielectric performance. This paper set out to highlight the effect of the particle interphase on electrical behaviour of nanodielectrics. First, the idea of particle interphase was illustrated and some of the already published experimental results were analyzed in accordance with this model. Additionally, experimental work designed to investigate the influence of the particle interphase was performed. In order to focus on the influence of the particle interphase, the other factors, mentioned above, were neutralized as much as possible by carefully choosing matrix material, particle material and processing conditions. 


\section{Particle Interphase Model (PIM)}

A nanoparticle has at least one dimension that falls within the nanoscale. As the dimensions reach the nanoscale, they approach the atomic scale where the number of the atoms forming such dimensions comes to be relatively countable and hence the bulk properties gradually start to dilute or disappear. For example, the electronic band structure, which consists of bands of energy states separated by band gaps, begins to convert into discrete energy levels when the particle size is less than $10 \mathrm{~nm}$ [21]. This may change the colour of materials with band gap in the visible light [22]. Other properties may behave in a similar way and become size-dependent [3, 33]. Even for larger dimensions, the outer layer atoms near the surface behave in a similar fashion. In fact, the classical macroscopic properties for a point in the bulk of a material are obtained by integrating over infinite (in an atomic scale) volumes around this point [22]. For example, point 1 in Figure 1 would give the macroscopic properties of the bulk of phase A if the dimensions of the particle A are considered atomically infinite. On the other hand, point 2 on the surface, in Figure 1, has a non-centro-symmetry nature and its behaviour may be affected by the existence of phase B. Similarly, atoms located a few layers under the surface, i.e. point 3 in Figure 1, will be similarly affected by the presence of phase B. The same thing is applicable to points 4 and 5 in phase $\mathrm{B}$. The properties at these points may be different from the properties of the bulk of both A and $\mathrm{B}$ and this region is what is meant by the terms interphase region or interaction zone. The thickness of the interphase depends on the property being considered and at what point the "infinite limit" is reached. In its turn, the infinite limit depends on the molecules size or molecules mass. In Figure 1, Phase A represents the nanoparticle and phase B represents the polymer matrix and, conventionally, it has been suggested that interaction-induced interphases primary affect the behaviour of the composite system through changes in the local structure and dynamics of the polymer REF: Barnardo et al. Computational Materials Science 118 (2016) 32-46. In the area of dielectrics the so-called multicore model was proposed by Tanaka [22] to explain many phenomena [23], but without proposing any mechanistic details. Nevertheless, it has recently been suggested that where electrical properties are concerned, the local density of states is of critical importance $[24,25]$ and, in this regard, it is possible that the structure of the nanoparticle close its surface may also be of importance. Indeed, the importance of interfacial states and their control is a well understood issue in many areas of electronics [26]. In this case, interphases that span the interface and include both components could be important; such a situation is represented schematically in Figure 1 in terms of a polymer interphase and a particle interphase). Although the interphase is depicted in Figure 1 to extend in phases A and B to a similar extent, this is purely for illustrative purposes and we accept that this is unlikely to be the case in practice; the interphase inside the particle's boundary (phase A) is expected to be less extensive than the interphase in the surrounding polymer (phase B). Additionally, in this figure, the boundary surface between the particle and the matrix is called "the interface", which we consider as the abrupt chemical boundary between the polymer and the nanoparticle and which, therefore, fails to consider structural, compositional and dynamical variations as this boundary is approached. 


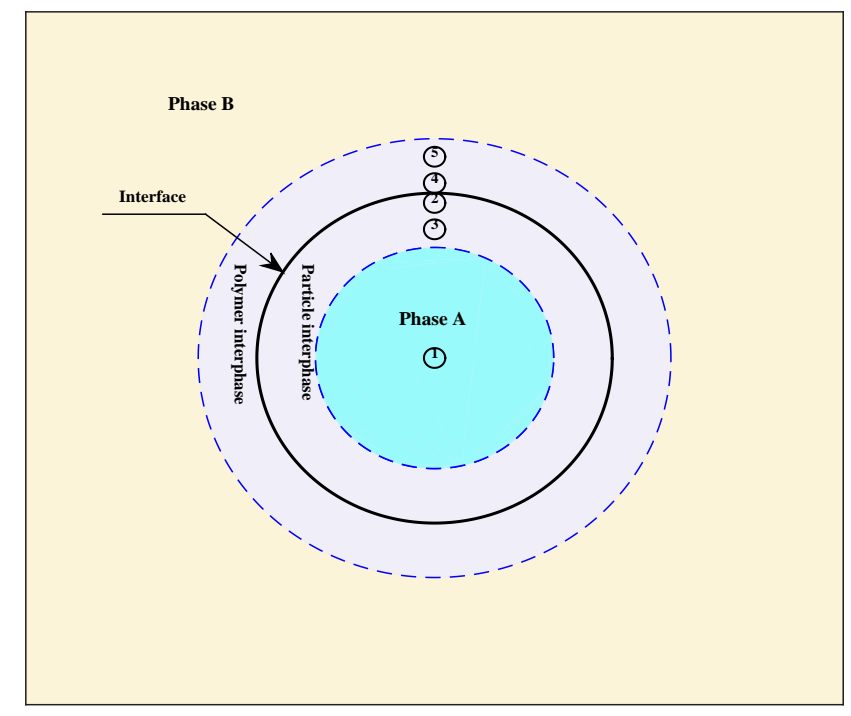

Figure 1. The interphase between two phases A and B (not to scale).

Due to their large surface area, nanoparticles contain a high concentration of defects on their surfaces. These defects could be due to the presence of foreign atoms on the surface, surface geometrical irregularities or the non-centro-symmetry nature (coordinative unsaturation) of the molecules near the surface of the particle [33]. As a result of these defects, new localized energy levels in an otherwise forbidden band are generated. The generated energy levels may lie in the band gap between the valence and the conduction bands and represent charge traps (localized states in the band gap). Upon the availability of mobile charges, i.e. electrons/holes in the conduction/valence band, they prefer to fill these traps rather than staying in the conduction/valence band [34]. Once it filled the trap, it is difficult for the electron/hole to return to the conduction/valence band, particularly if the trap is deep enough, although it can move to similar traps around the particles, if these are close enough [34]. Through this mechanism, charges may move through the particle interphase and if the particles are not far from each other, they may move from one particle interphase to another, as depicted in Figure 2. This might facilitate charge mobility in nanocomposites and degrade the electrical insulation properties. Obviously, such conduction in the band gap relies on the inter-particle distance and the trap density in the particle interphase [35]. For example, if these traps are deep and/or far from each other, the reverse could occur where the traps can capture the excited charge carriers and limit the conduction [16, 36, 37].

Alternatively, if the above-mentioned defects generate energy states that are deeper in the conduction/valence band, this might widen the band gap in the particle interphase region and constitute an energy barrier for the charge movement. As a result, the charge carriers will see the particle interphase, and consequently the whole particle, as an obstacle limiting their movement and increasing the length of their conduction paths as depicted in Figure 3. This might present a possible mechanism that results in improving the dielectric properties of the nanocomposites [36, 38]. 


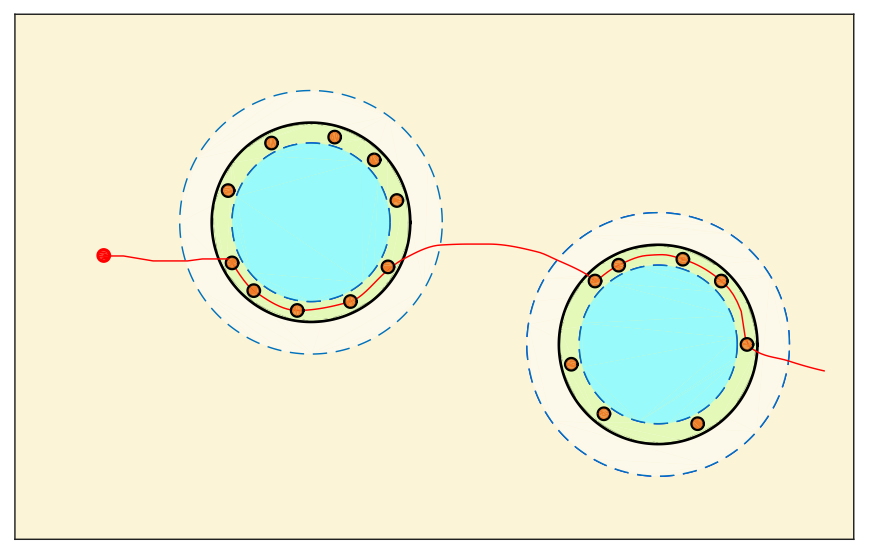

Figure 2. Possible mechanism for charge movement through the charge traps in the particle interphase (not to scale).

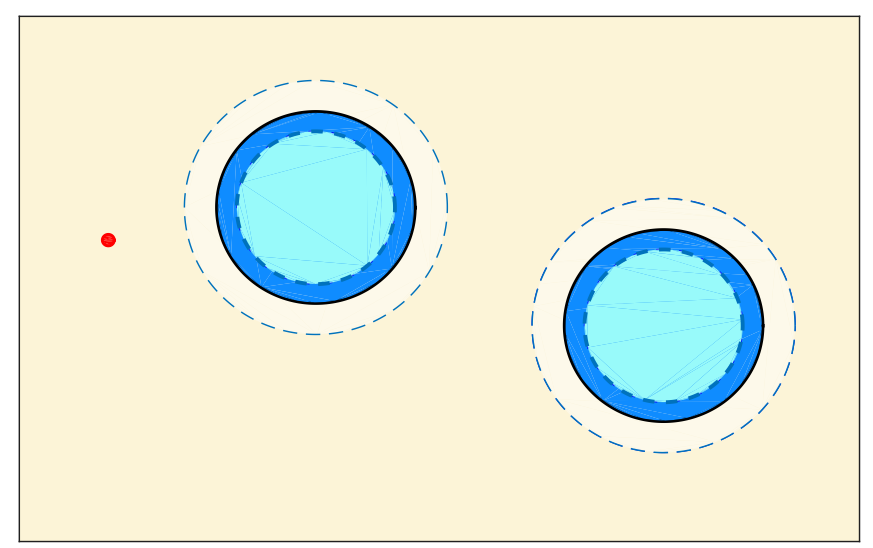

Figure 3. The particle interphase represents an energy barrier for charge movement (not to scale).

The crucial impact of the particle interphase on the dielectric behaviour could be pronounced in many experimental studies reported in the literature. The studies in $[39,40]$ investigate the effect of the addition of silica and hexagonal boron nitride ( $\mathrm{hBN}$ ) to an epoxy resin on the space charge dynamics and $\mathrm{AC}$ breakdown behaviour. The AC breakdown results correlate well with the space charge results, where the higher space charge accumulated in the silica epoxy nanocomposites leads to a lower AC breakdown strength. The reverse was observed in the boron nitride filled composites. It is expected that the surface of the hBN, due to its uniform structure, does not contain as many defects as exist on the surface of the silica, which means that the silica might contain more charge traps, which leads to the development of more space charge. Lau et al. [41] have shown that treating nano-silica particles with a silane coupling agent before introducing them into a polyethylene matrix reduces the formation of space charge and consequently increases the DC breakdown strength, compared with the untreated silica filled polyethylene. Complementary studies $[9,15]$ investigating the influence of varying the molecular length of the coupling agent, in an attempt to modify the polymer interphase, show that increasing the chain length of the silane functionalizer has no appreciable impact on the electrical properties. This suggests that the mechanism by which the particle treatment affects the electrical behaviour is not merely related to the interactions that occur in the polymer interphase. Interestingly, heat treatment of nano-silica which leads to the removal of surface hydroxyl groups, and thereby covering the surface with stable siloxane bridges, improves the dielectric performance of silica polyethylene nanocomposite [42]. These findings reveal that treating the nanoparticles in a way that modifies their surface chemistry (i.e. by replacing hydroxyl groups 
with silane molecules or removing hydroxyl groups thermally) may affect the localized states in the particle interphase region and that could be the reason behind the reduction in the space charge formulation, and subsequently, higher breakdown strength. A recent simulation study [43] considering the electronic density of states of magnesium oxide nanoparticles supports the above inference. The simulation has predicted that electronic traps are generated near the surface of the particle with a depth of $1.3 \mathrm{eV}$ when the particles are terminated with magnesium-oxygen bridges, $2.4 \mathrm{eV}$ when the particles are terminated with hydroxyl groups and $0 \mathrm{eV}$ (no traps) when the particles are terminated with a silane coupling agent. Although the simulation study is based on magnesium oxide, not silica, it is in line with the conclusion drawn from experimental observations that the electronic states near the surface of the particles are different from the states in the bulk and that they are very sensitive to the surface chemistry of the particles. Therefore, although the thickness of the particle interphase is expected to be very thin, it might have a decisive impact on the electrical properties of the nanodielectrics.

\section{Experimental}

\subsection{Materials}

An epoxy network was chosen to serve as a matrix. Epoxy is considered to be an amorphous material, therefore, the addition of the nanoparticles is not supposed to alter the morphology of the amorphous phase and, as a result, the effect of changing the morphology is neutralized. Specifically, the epoxy resin used here was DER 332; this is a diglycidyl ether of bisphenol-A (DGEBA) based system with an epoxide equivalent molar mass of $178 \mathrm{~g} \mathrm{~mol}^{-1}$, which was obtained from Sigma Aldrich. The hardener was Jeffamine D-230, which is an aliphatic diamine hardener with an amine hydrogen molar mass of $60 \mathrm{~g} \mathrm{~mol}^{-}$ 1, obtained from Huntsman. Based upon the above data, a theoretically ideal reaction stoichiometry of 1000 parts by mass of resin to 344 parts by mass of hardener can be defined. Both of these compounds were used as supplied.

Silicon nitride $\left(\mathrm{Si}_{3} \mathrm{~N}_{4}\right)$ amorphous nanopowder was utilized as the filler material. The manufacturer, Sigma Aldrich, states a spherical shape and a particle size $<50 \mathrm{~nm}$. The surface chemistry of the silicon nitride is characterized by the existence of amine and hydroxyl groups (oxynitride surface chemistry) [44, 45]. Previous work has shown that the amine hydrogens can chemically react with the epoxy groups in the resin [46, 47]. On one hand, this has the advantage of making silicon nitride inherently compatible with the matrix polymer, which should result in good particle dispersion and, thus, minimize the effect of particle agglomeration. On the other hand, this reaction will affect the effective resin/hardener stoichiometry. The latter effect was taken into account by adjusting the resin/hardener stoichiometry to compensate for the epoxy groups reacted with the amine groups on the silicon nitride nanofiller [47].

\subsection{Particle heat treatment}

To remove water molecules, which might be physically adsorbed on the surface, the nanopowder was dried at $200{ }^{\circ} \mathrm{C}$ for $10 \mathrm{~h}$ in a nitrogen environment. At $200{ }^{\circ} \mathrm{C}$, the surface hydroxyl or amine groups would not be affected [48]. This step was done to eliminate the possibility of forming water shells around the particles and, hence, offset the effect of water absorption.

In order to change the particle interphase, part of the silicon nitride nanopowder was heated at $1050{ }^{\circ} \mathrm{C}$ for $10 \mathrm{~h}$ in a nitrogen environment. This is expected to remove the active amine and hydroxyl groups, release any foreign atom on the surface and generate a system where the surface chemistry is dominated by stable siloxane bridges $[44,49]$. According to the discussion in section 2, this will affect the charge traps and the electron energy states within the particle interphase. While many other chemical processes have been used 
to modify the surface chemistry of nanoparticles [6, 50,51], calcination has the advantage of producing a relatively well-defined surface chemistry. For example, in silane surface treatment, it is impossible to ensure that the silane molecules replace all the surface hydroxyl groups [52]. Additionally, the silane molecules can condense with each other resulting in a complicated surface chemistry [53]. To discriminate between the two heat treatments, the powder which was heated at $200{ }^{\circ} \mathrm{C}$ is termed $\mathrm{SiN} 200$, whereas the powder heated at $1050{ }^{\circ} \mathrm{C}$ is termed SiN1050.

\subsection{Fourier Transform Infrared Analysis (FTIR)}

The surface chemistry of the nanoparticles was examined using a Perkin Elmer Spectrum GX Fourier transform infrared spectrometer operating in transmission mode. Suitable samples were prepared by mixing $1 \mathrm{mg}$ of the nanopowder with $150 \mathrm{mg}$ of ground potassium bromide (KBr) and pressing the resultant to form a $\mathrm{KBr}$ disc. The spectrum of each nanopowder was then collected in the range from 580 $\mathrm{cm}^{-1}$ to $7800 \mathrm{~cm}^{-1}$ with a resolution of $4 \mathrm{~cm}^{-1}$.

\subsection{Nanocomposite preparation}

Three sets of samples were prepared during the course of this study:

1- Unfilled epoxy: the resin and the hardener were mixed following the theoretical resin : hardener stoichiometric ratio of $1000: 344$ by mass, to give reference data for comparative purposes.

2- Nanocomposite containing 5 wt.\% of SiN200: as stated above, the silicon nitride surfaces contain amine groups, which may influence the effective resin/hardener ratio. From previous work [47], it can be estimated that the introduction of $5 \mathrm{wt} \%$ of this nano-powder should contribute the equivalent of 18 wt.\% of hardener to the system, such that the resin/hardener stoichiometric ratio should be offset by this amount. Consequently, the chosen resin/hardener ratio for this system was adjusted to 1000: $282(288=344 \times 0.82)$.

3- Nanocomposite containing $5 \mathrm{wt} . \%$ of SiN1050: since heat treatment at $1050{ }^{\circ} \mathrm{C}$ would be expected to remove the active amine groups on the nanoparticle surfaces, the resin/hardener ratio used for this sample was set at the theoretical optimum of $1000: 344$.

Table 1 summarizes the prepared samples along with relevant details; the procedures used to prepare these samples are detailed elsewhere [46, 47] and, consequently, are not repeated here, for the sake of brevity. All samples were stored under vacuum for at least two weeks prior to examination[54], in order to ensure that they were dry before any data were acquired.

Table 1. A summary of the investigated materials and their details.

\begin{tabular}{llll}
\hline Material code & $\begin{array}{l}\text { Resin : Hardener } \\
\text { mass ratio }\end{array}$ & Filler & Comments \\
\hline E/100H/0 & $1000: 344$ & 0 & Neat epoxy \\
E/82H/5SiN200 & $1000: 282$ & 5 wt.\% of SiN200 & Filled with dried $\mathrm{Si}_{3} \mathrm{~N}_{4}$ \\
$\mathbf{E} / \mathbf{1 0 0 H} / 5 \mathrm{SiN1050}$ & $1000: 344$ & $5 \mathrm{wt} \%$ of SiN1050 & Filled with calcinated $\mathrm{Si}_{3} \mathrm{~N}_{4}$ \\
\hline
\end{tabular}

\subsection{Material characterization}

To examine the nanoparticle dispersion within the epoxy, a cryo-fracture method was used, in conjunction with the scanning electron microscopy (SEM -JEOL JSM-6500F). Plaque samples $2 \mathrm{~mm}$ in thickness 
were cooled in a liquid nitrogen for $15 \mathrm{~min}$, then fractured before finally being sputter coated with gold to prevent charge accumulation during examination.

The thermal behaviour and the segmental dynamics of all the systems considered here were investigated using a Perkin Elmer DSC 7 differential scanning calorimeter (DSC). Two successive DSC scans from $30{ }^{\circ} \mathrm{C}$ to $150{ }^{\circ} \mathrm{C}$ at a heating rate of $10{ }^{\circ} \mathrm{C} \mathrm{min}{ }^{-1}$ were conducted on each specimen, of around $10 \mathrm{mg}$ in mass. The first DSC scan was used to erase the thermal history of each specimen, whereas data pertaining to the glass transition of the system were drawn from the second scan. Derived parameters include: the glass transition temperature $T_{g}$, defined as the point at which the change in the heat capacity is maximum; the change in the heat capacity over the glass transition $\left(\Delta C_{p}\right)$; the temperature range over which the glass transition occurred $\left(\Delta T_{g}\right)$, which provides an indication of the homogeneity of the material. These measurements were repeated on three separate specimens from each system, in order to determine measurement uncertainties.

In addition to examining the DSC glass transition, dielectric spectroscopy was used to probe changes in the segmental dynamics and the polar content brought about by inclusion of the nanofiller. For this, specimens $200 \pm 10 \mu \mathrm{m}$ in thickness were placed between two circular electrodes $30 \mathrm{~mm}$ in diameter and the dielectric response was measured at room temperature using a Solatron 1296 dielectric interface along with a Schlumberger SI 1260 impedance/phase gain analyzer. All samples were routinely sputter coated with gold on both sides to reduce any uncertainties associated with the contact between the sample and the dielectric spectrometer's electrodes.

Charge transport was evaluated by measuring the time dependence of the DC conductivity. For this, an electric field of $42 \mathrm{kV} \mathrm{mm}^{-1}$ was applied to specimens $200 \pm 10 \mu \mathrm{m}$ thickness and the resulting current was measured using a Keithley 6487 picoammeter. For each sample, one measurement was taken per one minute for two hours and, since the observed current was not found to vary significantly throughout the experiment, the quoted DC conductivity values simply correspond to the average of these 120 measurements. As for dielectric spectroscopy, these samples were again sputtered coated with gold electrodes prior to data being acquired, to minimize sample contact issues. In this case, $20 \mathrm{~mm}$ diameter circular sputtered electrodes were applied, to match the electrodes in the test cell. The sample temperature was controlled by conducting all measurements in a fan oven.

DC breakdown measurements were conducted by placing specimens $70 \pm 5 \mu \mathrm{m}$ in thickness between opposing $6.3 \mathrm{~mm}$ diameter steel ball bearing electrodes and increasing the applied voltage at a ramp rate of $100 \mathrm{~V} \mathrm{~s}^{-1}$ until breakdown occurred. The electrodes were replaced every 5 measurements, to avoid surface pitting from affecting the data. All breakdown data were acquired at room temperature $\left(23{ }^{\circ} \mathrm{C}\right)$ and a two-parameter Weibull function was employed statistically to analyze the results.

\section{Experimental results and discussion}

\subsection{Heat treatment and filler surface chemistry}

Comparing the mass of the nanopowder before and after heating at $200{ }^{\circ} \mathrm{C}$ showed that the nanopowder lost $\sim 1.6 \%$ of its initial mass during the process. Comparison of this result with comparable data obtained from a silica nanopowder, which when treated under the same conditions lost more than $16 \%$ of its weight [55], indicates that silicon nitride does not absorb much water when stored under ambient conditions. This is primarily ascribed to the surface of silicon nitride being partially covered by amine groups, which are less polar than the hydroxyl groups that dominate in silica. Furthermore, Fubini et al. 
[48] reported that the hydroxyl groups on silicon nitride have slightly less acidity than the corresponding ones on silica. Figure 4 shows FTIR data obtained from samples of nano-silicon nitride after thermal treatment at $200{ }^{\circ} \mathrm{C}$ and $1050{ }^{\circ} \mathrm{C}$, from which it is evident that, in both samples, the intensity of the small absorption peak at peak at $1633 \mathrm{~cm}^{-1}$ is comparable. Since this peak is associated with the presence of molecular water [56], its invariance between the two differently processed systems suggests that the two thermal treatments are equally effective at removing the physically adsorbed water.

Consider, first, the FTIR spectrum of SiN200. This contains a pronounced peak at $3140 \mathrm{~cm}^{-1}$, which can be assigned to $\mathrm{NH}$ vibrations $[45,57,58]$, together with another absorption centred at $3422 \mathrm{~cm}^{-1}$, which is related to the presence of $\mathrm{OH}$. The notable breadth of both of these peaks is an indicator of hydrogen bonded amine and hydroxyl groups, while the cumulative intensity implies a high concentration of both moieties [45, 58]. The sharp peak at $1400 \mathrm{~cm}^{-1}$ corresponds to the $\mathrm{Si}_{2}-\mathrm{NH}$ deformation mode [57], while the absorption between $750 \mathrm{~cm}^{-1}$ and $1150 \mathrm{~cm}^{-1}$ is due to two overlapping peaks, one at $965 \mathrm{~cm}^{-1}$, which is attributed to the backbone vibration of Si-N-Si bonds, and the other at $1075 \mathrm{~cm}^{-1}$, which is associated with the stretching vibration of Si-O-Si bonds (siloxane bridges) [57, 59]. The presence of this latter feature is indicative of a fraction of the particle surface having, effectively, been oxidized to silica [44, 57]. The weak absorption between $2800 \mathrm{~cm}^{-1}$ and $2900 \mathrm{~cm}^{-1}$ can be assigned to C-H stretching; a similar C-H absorption was previously reported by Li et al. [44], who suggested that this bond arises as a consequence of $\mathrm{SiN}_{2} \mathrm{H}$ reacting with atmospheric $\mathrm{CO}_{2}$. In conclusion, the FTIR spectra of SiN200 indicate a complex surface chemistry characterized mainly by the presence of $\mathrm{NH}$ and $\mathrm{OH}$ groups, together with some additional impurities.

Calcining at $1050{ }^{\circ} \mathrm{C}$ (SiN1050) results in a significantly different spectrum (see Figure 4). First, the disappearance of the peaks at $3140 \mathrm{~cm}^{-1}$ and $1400 \mathrm{~cm}^{-1}$ indicates the removal of the $\mathrm{NH}$ groups. Furthermore, the peak at $1075 \mathrm{~cm}^{-1}$ (siloxane bridges) becomes stronger, whereas the peak at $965 \mathrm{~cm}^{-1}$ becomes very weak. This implies an increase in the Si-O-Si concentration accompanied by depletion of some of the Si-N-Si bonds [44]. The weakness of Si-N-Si absorption $\left(965 \mathrm{~cm}^{-1}\right)$ and the absence of absorption peaks associated with amine groups implies that this absorption originates from the Si-N-Si in the bulk of the particles, rather than on the surface, particularly since the spectra were collected in transmission mode [44]. Therefore, the spectra of SiN1050 suggest that the surface of the particles is predominantly covered by a silica layer. Additionally the presence of a peak at $813 \mathrm{~cm}^{-1}$, assigned to siloxane bridges, and the absence of any peak at $\sim 951 \mathrm{~cm}^{-1}$, which is normally observed for silica and assigned to Si-OH groups [56], suggest that the silica layer on the particle surface is terminated with siloxane bridges rather than silanol groups. Comparable conclusions concerning the surface chemistry of silicon nitride powder heated above $1000{ }^{\circ} \mathrm{C}$ have been reported elsewhere [44].

To provide further supporting evidence concerning the presence of siloxane bridges on the surface of our calcined nano-silicon nitride, complementary FTIR data were also obtained from a silica nano-powder, which was subjected to the same heat treatment at $1050^{\circ} \mathrm{C}(\mathrm{SiO} 1050)$. The resulting data are compared with the FTIR spectrum of SiN1050 in Figure 5, which reveals considerable similarities. However, as would be expected, the Si-N-Si absorption at $965 \mathrm{~cm}^{-1}$ is absent in the spectra of SiO1050, which is due to the absence of this bond in the bulk of the silica powder. In summary, we interpret the FTIR data discussed above as demonstrating the following. First, both imposed thermal treatments are effective in removing physically adsorbed water from the as-received nanosilica. Second, while treatment of $200{ }^{\circ} \mathrm{C}$ retains the initial oxynitride surface layer, treatment at the higher temperature converts this to one that is predominantly silica (terminating with siloxane bridges), such that these nanoparticles can be considered 
to have a silicon nitride/silica core/shell structure. Third, these findings are consistent with published literature.

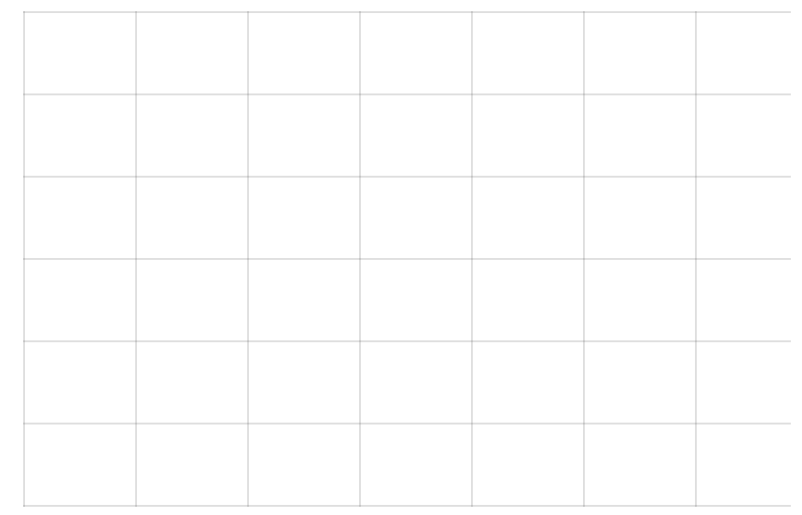

Figure 4. FTIR spectra for SiN200 and SiN1050.

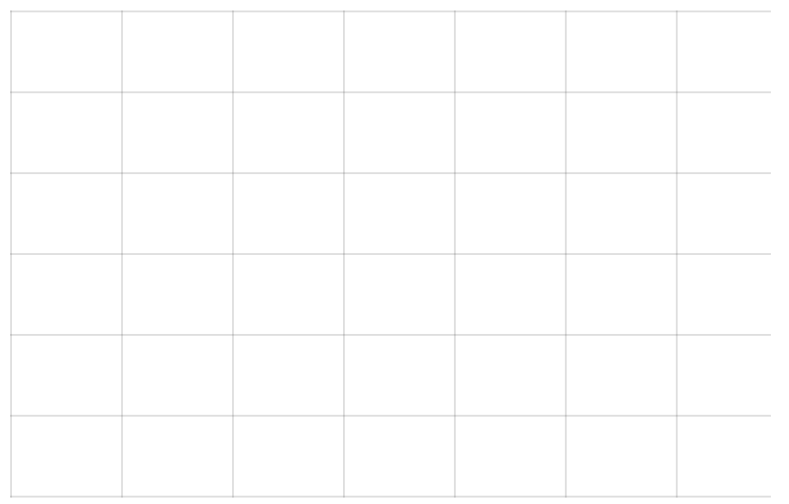

Figure 5. FTIR spectra for SiN1050 and SiO1050.

\subsection{Particle Dispersion}

Representative SEM images of all the systems considered here are shown in Figure 6. The micrograph of the unfilled epoxy (see Figure 6a) shows a typical amorphous structure that is largely devoid of any surface features, which is consistent with results reported elsewhere [60,61]. The image of the SiN200 filled epoxy, Figure 6b, reveals a surface after cryofracture, which is quite distinct from that shown in Figure 6a, as is often reported for systems containing nanoparticles. This results from interactions between the distribution of nanoparticles in the system leading to crack pinning and the consequent formation of conventional fractography features [60,61]. A higher magnification image of the same system is shown in Figure 6c; in this, details of the fractography features and the granularity of the gold coating can both be seen, together with circular entities. These are of commensurate dimensions to the specified size of the nanoparticles and, as such, we interpret that arrowed structures in terms of the included nanoparticles. No large particle agglomerations can be seen, albeit that there are occasional small agglomerations, where a few nanoparticles are clustered together. For the SiN1050 filled epoxy, Figure 6d shows a somewhat rougher surface texture, which may be related both to interfacial effects and/or the degree of particle dispersion. A higher magnification image of this system, Figure 6e, reveals no evidence of gross changes in individual nanoparticle morphology, but does suggest the existence of particle clusters are formed from 
loose particle agglomerations. The difference in the agglomeration state of the SiN200 and SiN1050 nanoparticles may be a consequence of differences in surface chemistry leading to differences in nanoparticle/matrix interactions [62-64]. As the FTIR spectra showed, SiN200 has a high concentration of surface amine groups, which can form strong interactions with the epoxy matrix, whereas the SiN1050 is covered with siloxane bridges, which cannot form such strong interactions with the polar epoxy matrix.

(a)

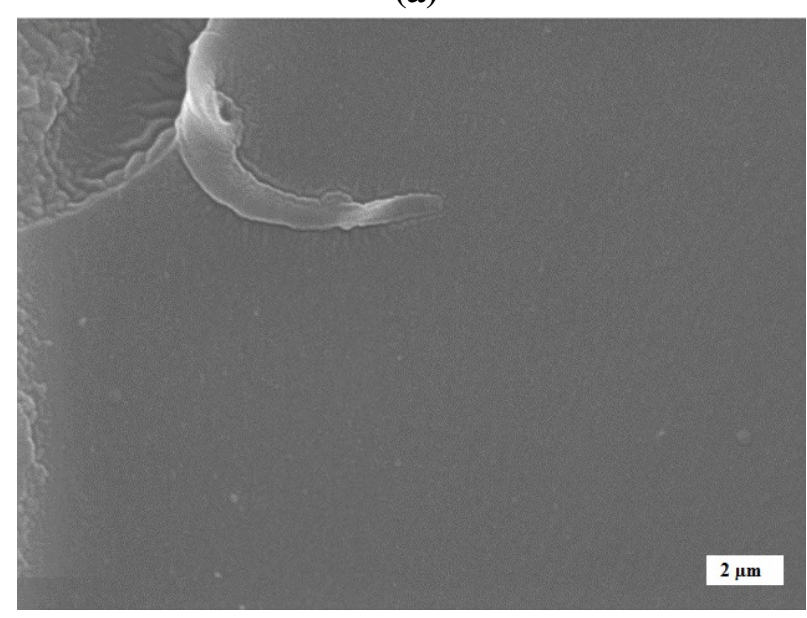

(b)

(c) 


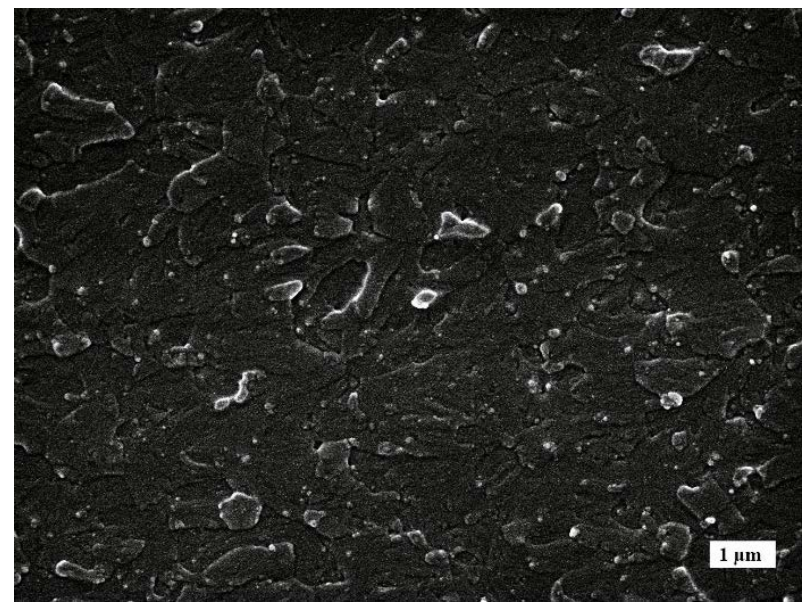

(d)
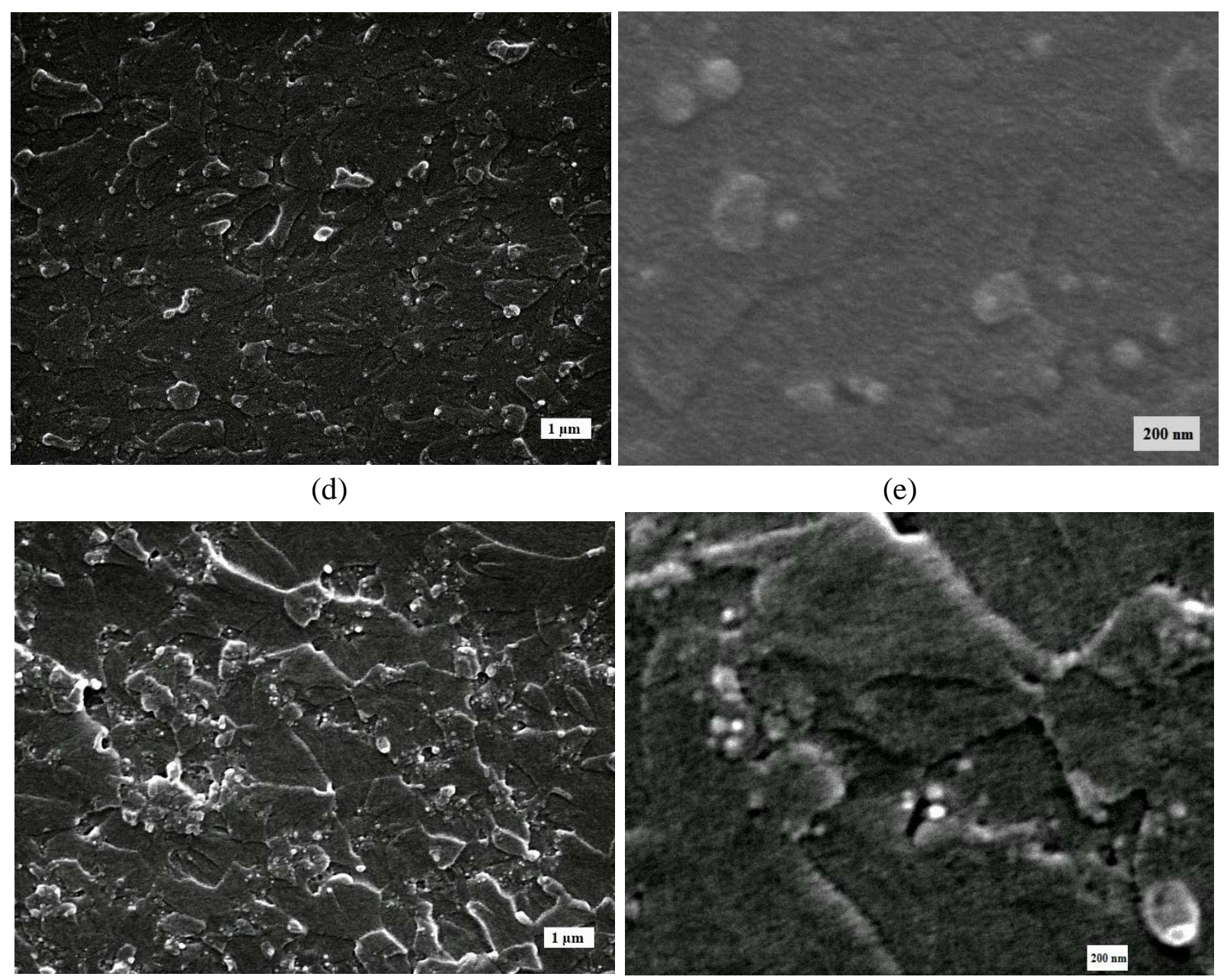

(e)

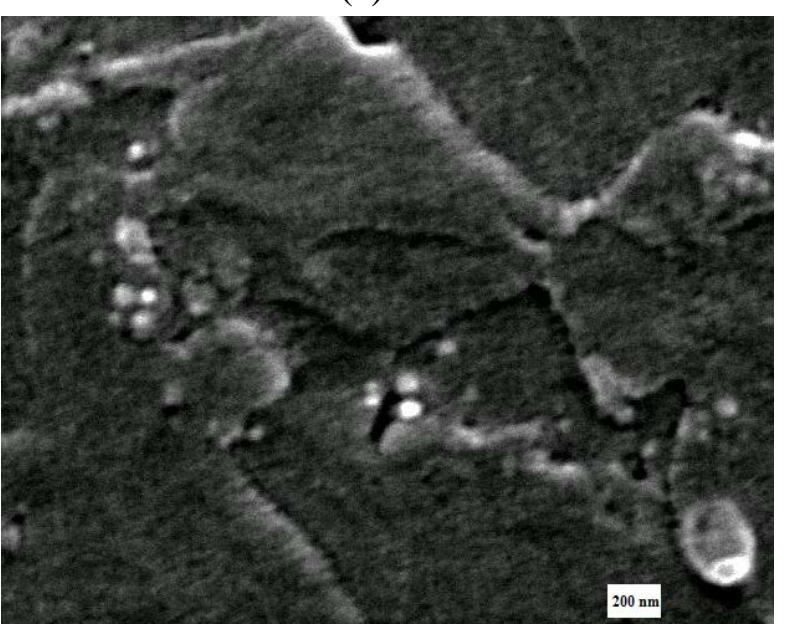

Figure 6. SEM images for (a) sample E/100H/0, (b) sample E/82H/5SiN200, (c) sample E/82H/5SiN200 high magnification image, (d) sample E/100H/5SiN1050, and (e) sample E/100H/5SiN1050-high magnification image.

\subsection{Glass Transition and Molecular Dynamics}

The FTIR data presented above demonstrate that the imposed thermal treatments result in changes in nano-silicon nitride surface chemistry and, specifically, that calcination at $1050{ }^{\circ} \mathrm{C}$ results in removal of the surface amine character that can affect the epoxy curing reaction. Figure 7 contains representative DSC traces for all samples, which reveal the influence of changing the material composition on the DSC glass transition. Derived parameters are summarized in Table 2. 
Figure 7. DSC traces for the three samples; the circles superimposed on the curves indicate $T_{g}$.

Table 2. Glass transition parameters for the three samples, the confidence interval is based on $95 \%$ probability.

\begin{tabular}{llll}
\hline sample code & $\boldsymbol{T}_{\boldsymbol{g}}\left({ }^{\mathbf{0}} \mathbf{C}\right)$ & $\Delta \boldsymbol{C}_{\boldsymbol{p}}\left(\mathrm{J} \mathrm{g}^{-\mathbf{1}}{ }^{\mathbf{0}} \mathbf{C}^{-\mathbf{1}}\right)$ & $\Delta \boldsymbol{T}_{\boldsymbol{g}}\left({ }^{\mathbf{0}} \mathbf{C}\right)$ \\
\hline $\mathbf{E} / \mathbf{1 0 0 H} / \mathbf{0}$ & $88.2 \pm 2.3$ & $0.31 \pm 0.026$ & $7.66 \pm 1.8$ \\
$\mathbf{E} / \mathbf{8 2 H} / \mathbf{5 S i N 2 0 0}$ & $87.5 \pm 2.3$ & $0.316 \pm 0.026$ & $7.1 \pm 1.8$ \\
$\mathbf{E} / \mathbf{1 0 0 H} / \mathbf{5 S i N 1 0 5 0}$ & $88.5 \pm 2.3$ & $0.309 \pm 0.026$ & $6.79 \pm 1.8$
\end{tabular}

From the above data, it is evident that the DSC glass transition in all three systems is comparable, despite the modified epoxy/hardener ratio used in E/82H/5SiN200. Recently, we have shown for the same epoxy/hardener system that, in the absence of any compensating effect, modifying the stoichiometry to this degree would result in a depression in $T_{g}$ of $\sim 22{ }^{\circ} \mathrm{C}[31,47]$. The fact that no comparable reduction in $T_{g}$ is seen here provides qualitative validation of the active role played by the surface amines groups in $\mathrm{E} / 82 \mathrm{H} / 5 \mathrm{SiN} 200$ in the epoxy cure reaction and quantitative verification of the assumed stoichiometric perturbation provided by adding $5 \mathrm{wt}$.\% of SiN200. A necessary corollary of this is that calcining the nano-silicon nitride in E/100H/5SiN1050 has indeed removed the amine surface functionality, such that it exhibits the same $T_{g}$ value as E/82H/5SiN200, despite the different matrix resin stoichiometry.

The fact that all three systems exhibit equivalent glass transitions indicates that, within experimental uncertainties, all three systems undergo identical glass transition processes. That is, the main chain dynamics of the polymeric matrix in all of these samples are equivalent, such that no variations can be detected by this characterization method. This observation further suggests that in both nanocomposite systems, whether the nanoparticles strongly interact (as would be expected in E/82H/5SiN200) or do not interact (as would be expected in $\mathrm{E} / 100 \mathrm{H} / 5 \mathrm{SiN} 1050$ ) with the epoxy matrix, the nanofiller does not induce an observable confined or loose layer around themselves. This, in turn, implies either the absence of such layers, as was reported by [25], or that the thickness of such layers are too thin $(<\sim 1 \mathrm{~nm})$ to be detected out of the experimental uncertainties at the filler loading level considered here ( $5 \mathrm{wt} . \%$ or 1.65 vol.\%) [27].

Dielectric spectra obtained from the three samples are presented in Figure 8, which show that the real part of the permittivity $\left(\epsilon_{r}^{\prime}\right)$ is comparable in all three systems. The evident, slight differences in the absolute magnitude of $\epsilon_{r}^{\prime}$ (see Figure 8a) are within the experimental uncertainties; such variations often result 
from errors related to determining the true samples thickness or in producing electrode layers that are exactly $30 \mathrm{~mm}$ in diameter and precisely aligned with one another on both sides of the samples. That is, such variations in $\epsilon_{r}^{\prime}$ stem from uncertainties in the input parameters used to transform measured sample capacitances into permittivity values. The imaginary permittivity data shown in Figure 8b contain a typical $\beta$ relaxation which, in all three systems, is of comparable strength. It has been shown $[65,66]$ that this relaxation is associated with the hydroxyether groups that result from the reaction of the resin and the hardener and, consequently, its strength should be proportional to the crosslinking density present in the system. Previously, we have shown that reducing the hardener content in the absence of any compensating effects results in a sharp reduction in its $\beta$ relaxation [31]. Consequently, the data shown in Figure 8 further demonstrate that the crosslink density in all three systems is equivalent, which is in consistent with the DSC glass transition results discussed above.

Nevertheless, to investigate molecular processes further, the temperature dependence of the dielectric response of the three systems was considered and the resulting raw data obtained from $35{ }^{\circ} \mathrm{C}$ (i.e. well below the $T_{g}$ as established with DSC) to $110^{\circ} \mathrm{C}$ (i.e. well above the DSC $T_{g}$ ) are shown in Figure 9 . From these results, it is evident that the dielectric response of the E/100H/0 and E/100H/5SiN1050 systems are equivalent at all temperatures, but that the behaviour of E/82H/5SiN200 is very different, in that marked low frequency losses becomes increasingly evident as the temperature increases. While it is tempting to associate this directly with the change in matrix formulation resulting in a change in network architecture and consequent modified molecular dynamics, we believe that this is not the case here. Analysis of the frequency dependence of the imaginary component of the relative permittivity of all three systems well above $T_{g}$ demonstrates that this parameter varies with the reciprocal of the frequency. That is, the observed dielectric losses are associated with charge transport processes. As such, it is possible to subtract this contribution from the imaginary dielectric spectra, to reveal the underlying molecular dynamics: Figure 10 shows results obtained following such data manipulation, from which, two significant factors emerge. First, it is evident that both the strength and the frequency of the $\alpha$ relaxation in all three systems are equivalent at both $100{ }^{\circ} \mathrm{C}$ and $110^{\circ} \mathrm{C}$. Second, additional dielectric relaxation process have previously been observed in nanocomposite systems and related to molecular relaxations in the vicinity of nanoparticle surfaces. Since the shape of the $\alpha$ relaxation peak in all three of our systems is comparable, we conclude:

- The $\beta$ relaxation that can be seen at the upper end of the frequency range at room temperature is equivalent in the three observed systems;

- The $\alpha$ relaxation is equivalent at all the temperatures considered here in all three systems;

- No additional features are seen in either nanocomposite system that can be associated with interphase regions within the polymer.

- Therefore, no evidence exists for the nanoparticle-induced changes to molecular dynamics in the specific material systems considered here

The latter is not intended as a generally applicable conclusion, but for seems applicable to amorphous systems like the epoxy resin used in this study. Finally, having considered the molecular dynamics after removal of the charge transport contribution to the dielectric loss, it is worth examining the temperature dependence of charge transport itself. To do so, we need to estimate the contribution of the $\alpha$ relaxation at each temperature and then subtract this from the dielectric spectra in order to reveal the residual contribution associated with charge transport. The $\alpha$ relaxation at 100 and $110^{\circ} \mathrm{C}$ can be determined from Figure 10 and, consequently, at temperatures $\leq 90^{\circ} \mathrm{C}$, the contribution to the overall dielectric loss can be estimated by simply shifting the results shown in Figure 10 to the left, depending on the temperature 
[REF: Jonscher, Dielectric Relaxation in Solids]. For example, the left shift of the $\alpha$ relaxation when the temperature decreases from $100{ }^{\circ} \mathrm{C}$ to $90{ }^{\circ} \mathrm{C}$ is considered the same as the shift of this relaxation when the temperature decreases from $110{ }^{\circ} \mathrm{C}$ to $100{ }^{\circ} \mathrm{C}$. Figure 11 shows the obtained results, which reveal a low frequency element whereby the imaginary permittivity varies with the reciprocal of frequency - as would be anticipated for a charge transport process. This, together with a relatively small increase in high frequency losses, we associate with remnants of the $\beta$ relaxation. Mathematically, this data analysis process equates to the standard use of a shift factor whereby, in the case of a specific relaxation, the frequency corresponding to maximum loss is commonly determined as a function of temperature, and used in an Arrhenius analysis to estimate the activation energy of the associated process. In the case of charge transport, it is clearly not possible to follow such a procedure exactly, but it is possible to determine the equivalent shift factor in terms of the variation with temperature of the frequency corresponding to particular value of imaginary permittivity. The result of such an analysis are shown in Figure 12; in this case, $\epsilon_{r}^{\prime \prime}$ was arbitrarily chosen for ease of analysis, but the use of other values did not affect the general conclusion. This figure suggests that for the three systems two different mechanisms for charge transport occur. One dominates at temperatures higher than $T_{g}$, the other at temperatures lower than the $T_{g}$. At temperatures above the $T_{g}$, the major charge transport contribution in such epoxy systems can be attributed to ionic conductivity [23]. The parallelism of the three Arrhenius lines of the three systems indicates comparable conduction activation energy, which consequently implies comparable network structure that similarly interacts with the ionic movement in the three systems. As the temperature decreases below $T_{g}$, the epoxy network enters its glassy state, which limits ionic transport. Thus, at temperatures below $T_{g}$, an alternative charge transport process becomes dominant. While it is not possible to unambiguously identify this from the data presented, we suggest that this may be electronic in origin. Again, Figure 12 shows that the Arrhenius lines for the three systems are parallel. Whereas, this result suggests that at temperature below $T_{g}$, the charge transport in the three systems is equivalent, it does not explain why the conduction of sample E/82H/5SiN200 is greater. More discussion concerning DC conduction is given in the next section.

We argue that the totality of the evidence presented above leads to a number of conclusions, which are applicable to the systems considered here.

- First, that SiN200 should be considered both as a nano-filler and as a reactant and that this latter effect was properly accounted for in E/82H/5SiN200 by changing the epoxy to hardener ration used to formulate the matrix.

- Second, the presence of neither nanofiller leads to any detectable alteration in dielectric relaxation processes as a consequence of the introduction of the nanoparticles. This is in contrast to the work of Hosier et al. [54], who observed that the addition of silicon nitride into a polyethylene matrix results in a low frequency relaxation that was progressively displaced to higher frequencies when the nanocomposite material was exposed to water. The absence of any additional relaxation in the nanocomposites investigated here therefore (a) implies that no significant water shells are present around the nanoparticles $[11,12]$ and (b) indicates that any regions close to the nanoparticle surfaces in which the molecular dynamics of the polymer chains are modified are so limited in extent as not to be detectable.

- Third, we suggest that by varying the nanofiller thermal treatment and appropriately compensating for the influence that the nanofiller surface chemistry has on the epoxy cure reaction, we have generated three systems which differ only in terms of their intrinsic nanofiller surface chemistry/structure. In this case, the influence of the nanofiller surfaces on bulk electrical properties should be directly revealed: 
E/100H/0 contains no nanoparticle surfaces; in E/82H/5SiN200, nanoparticle surfaces are based on un-relaxed silicon oxynitride chemistry/structure; in E/100H/5SiN1050 nanoparticle surface are based on thermally-relaxed silica (siloxane bridges).

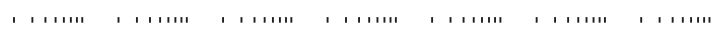

Figure 8. Dielectric response, (a) real part of the relative permittivity and (b) imaginary part of the permittivity. 


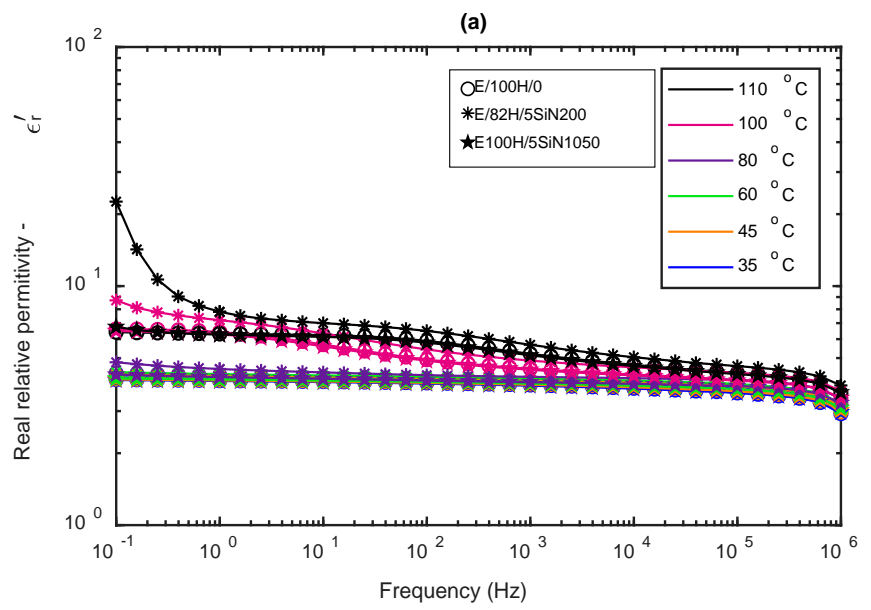

(b)

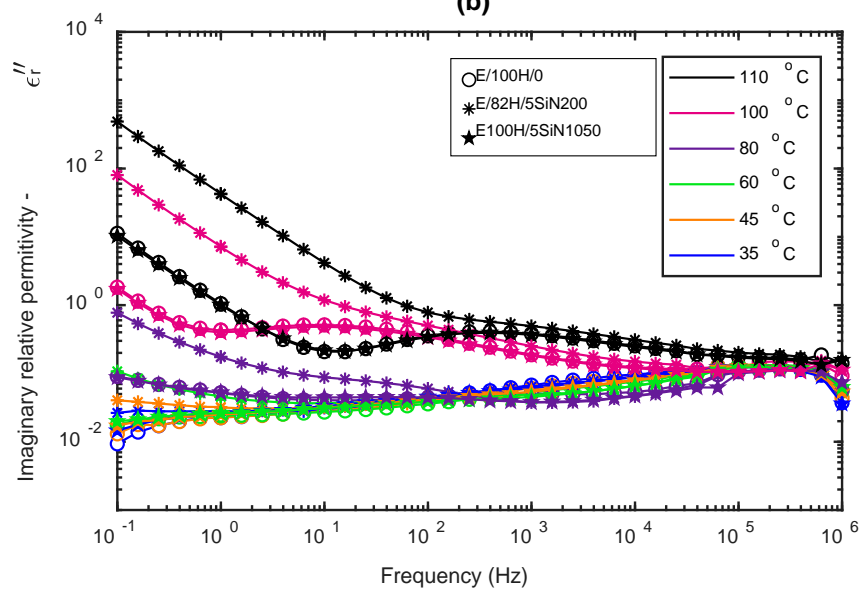

Figure 9. Dielectric spectra versus temperature, (a) real part of the relative permittivity and (b) imaginary part of the permittivity.

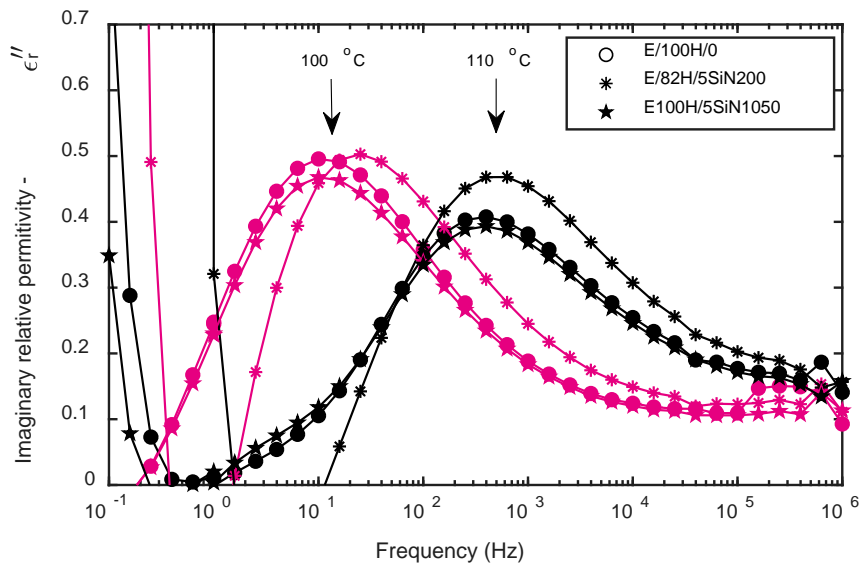

Figure 10. Imaginary part of the permittivity after subtracting the contribution of DC conductivity at 100 and $110^{\circ} \mathrm{C}$. 


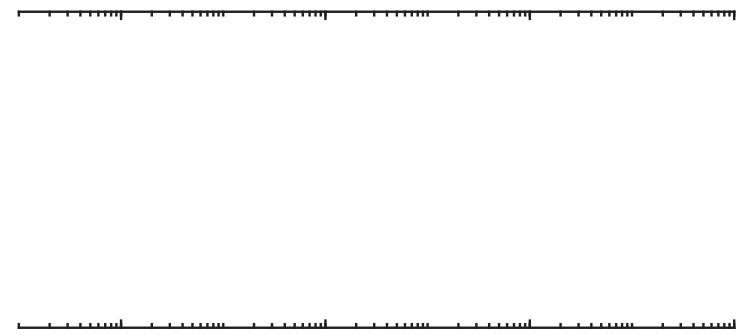

Figure 11. Imaginary part of the permittivity after subtracting the contribution of the $\alpha$ relaxation at selected temperatures for (a) E/100H/0, (b) E/82H/5SiN200 and (c)E/100H/5SiN1050.

$\longrightarrow+1+1,1+19$

Figure 12. Effect of material formulation on the temperature dependence of charge transport, represented as an Arrhenius-type plot. 


\subsection{Conductivity and Breakdown Strength}

The time dependence of the DC conductivity at $45{ }^{\circ} \mathrm{C}$ of the three systems considered here is shown in Figure 13. The reduced scattering in the data obtained from sample E/82H/5SiN200 compared with the data obtained from the other samples is a simple consequence of the higher signal to noise ratio in these data, which stems directly from the higher current that flows through this sample. From these data, it is evident that any time dependence is weak, thereby validating the decision to take a simple average of all the measured data points to represent the DC conductivity of each system. Praeger et al. [15] reported time dependent conductivity data for nanosilica filled polyethylene, in which an initial decrease in conductivity with time was followed by a period in which the conductivity increased, an effect that they ascribed to dynamic water absorption effects. The slight increase in conductivity with time for E/82H/5SiN200 that is evident in Figure 13 at longer times may have a similar origin but, in this case, the effect is relatively minor compared to the marked differences that exist between this and the other systems. Repeating these measurements at different temperatures results in the data shown in Figure 14, which shows the mean of all the acquired conductivity values plus error bars, which represent the $95 \%$ confidence bounds derived from each data set. From this figure, it is clear that the DC conductivity of the SiN200 filled system is significantly greater than that of the other two systems at all the temperatures considered here. The SiN1050 filled epoxy has a slightly lower DC conductivity than the unfilled epoxy.

This trend in the DC conductivity distinctly correlates with the DC breakdown results shown in Figure 15 and Table 3. Since the polymer matrix in the three materials does not exhibit any significant variations, as demonstrated by the glass transition results and the dielectric response, these variations in the DC conductivity and breakdown strength cannot be related to variations in the epoxy network dynamics or structure. Additionally, a previous work [31] has concluded that the electrical behaviour of the same epoxy matrix does not correlate with the network structure or crosslinking density. Therefore, theses variations in electrical properties must be associated with the nanoparticles. Regarding the particle dispersion, the SiN200 dispersion is much better than the SiN1050 dispersion and, hence, the dispersion factor would advise that the system filled with SiN200 has a better electric performance [17], which is not the case. Accordingly, the particle dispersion is not a decisive factor in determining the charge transport dynamics in these systems. Praeger et al. [42] studied the effect of calcinating a silica nanofiller at 1050 ${ }^{\circ} \mathrm{C}$ before introducing it into a polyethylene matrix. They found that the calcinated silica resulted in a considerable increase in the DC breakdown strength, while the as-delivered silica, without any heat treatment, caused a substantial reduction in the DC breakdown strength; no other heat treatment was tried. The authors referred this behaviour to the water shells that surround the hydrophilic as-delivered silica and evaporate upon heating the particles at $1050{ }^{\circ} \mathrm{C}$. Water molecules are highly polar and can be auto-ionized (i.e. producing $\mathrm{OH}^{-}$and $\mathrm{H}_{3} \mathrm{O}^{+}$ions), leading to higher charge transport [12, 67]. In the current investigation, care was taken to remove such water layers by heating the particles at $200{ }^{\circ} \mathrm{C}$ and drying the samples at vacuum for $>14$ days. Additionally, as was discussed when analyzing the dielectric response, no evidence has been identified for the presence of water shells around the SiN200 particles. Returning to the FTIR results, the surface of SiN200 particles has complex chemistry, is partially oxidized to silica, and contains many defects as indicated by detecting carbon and hydrogen atoms. Beside the amorphous structure of the particles, this surface chemistry suggests disordered band gap in the particle interphase with a high concentration of localized electronic states. As was discussed earlier, such particle interphase might increase the charge transport, as was illustrated by Figure 2, and thus it may account for the higher DC conductivity and lower DC breakdown strength observed for this sample. On the other hand, the 
SiN1050 is covered by a silica layer terminating with stable siloxane bridges without NH or OH groups. Such surface chemistry indicates particle interphase with a lower concentration of localized electronic states. Indeed, Sameshima et al. found that changing the surface chemistry of silica films into Si-O-Si network significantly reduces the density of trap states [68]. Furthermore, it is known that the silica has a considerable larger band gap than the silicon nitride [69], which implies a particle interphase with wider band gap. Consequently, the particle interphase, in this case, might work as an energy barrier that scatters charge carriers as was proposed by Figure 3. Such particle scattering effect has already been cited elsewhere [36, 38] as a mechanism for improving dielectric properties of nanocomposites and, thus, the PIM model presents a possible explanation for the origin of this effect.

Figure 13. DC conductivity measurements at $45^{\circ} \mathrm{C}$ and electric field of $42 \mathrm{kV} \mathrm{mm}{ }^{-1}$.

Figure 14. Average of DC conductivity for all samples at different temperatures and electric field of 42 $\mathrm{kV} \mathrm{mm}^{-1}$, the error bars indicate the $95 \%$ confidence bounds of the average. 
Figure 15. DC breakdown measurements at room temperature.

Table 3. Weibull parameters derived from the DC breakdown measurements for all samples, the uncertainties are based on the $95 \%$ confidence bounds.

\begin{tabular}{|c|c|c|c|}
\hline Sample code & $\mathrm{E} / \mathbf{1 0 0 H} / \mathbf{0}$ & E/82H/5SiN200 & E/100H/5SiN1050 \\
\hline $\begin{array}{l}\text { Weibull scale parameter } \\
\left(\mathrm{kV} \mathrm{mm}^{-1}\right)\end{array}$ & $646 \pm 18$ & $472 \pm 12$ & $660 \pm 17$ \\
\hline Weibull shape parameter & $19 \pm 6$ & $22 \pm 7$ & $20 \pm 6.8$ \\
\hline
\end{tabular}

\section{Conclusions}

In this study, we propose dividing the interphase region between the nanoparticles and the polymer into particle interphase and polymer interphase. Since most reported work has concentrated on the polymer interphase, the emphasis here was for highlighting the importance of the particle interphase. The study demonstrated that the particle interphase has a critical influence on the electrical properties of nanodielectrics, which might be more crucial than the polymer interphase. This may be attributed to that the particle material has higher density and, consequently, any perturbations in the particle interphase will lead to modifying the electronic density of states and, therefore, affect the electronic dynamics in this region and, ultimately, in the whole nanodielectric system.

The electrical properties are essentially affected by the dynamics of charge carriers, which could be as small as electrons, and therefore, the behaviour of these properties is not necessarily correlated with the dynamics of larger constituents, i.e. dynamics of the polymeric segments or chains. The experimental results clearly support such conclusion, where all the samples exhibit similar molecular dynamics but significantly different charge dynamics and consequently, electrical performance. Therefore, designing nanodielectrics might need deeper and more analytical approach than the currently empirically-dominated approach. Such approach should consider the relative location of the conduction and valence energy levels between the filler and matrix materials. These levels can be engineered by properly selecting the suitable filler/matrix combinations. Furthermore, the variations that may occur in these levels in the interphase regions should be considered, since they can lead to a significant impact on the dielectric performance of nanodielectric systems. 


\section{Acknowledgments}

The authors would like to thank Dr Suvi Virtanen and Dr Matt Praeger for inspiring discussions and also for their training on FTIR spectroscopy measurements. The authors also acknowledge Mr Nick White for his assistance with using the high temperature furnace at the Optoelectronics Research Centre.

\section{References}

[1] T.J. Lewis, Nanometric dielectrics, IEEE Trns. Dielectr. Electr. Insul. 1(5) (1994) 812-825, DOI: 10.1109/94.326653.

[2] J.K. Nelson, J.C. Fothergill, L.A. Dissado, W. Peasgood, Towards an understanding of nanometric dielectrics, IEEE Conf. Electr. Insul. Dielectr. Phenom. (2002) 295-298, DOI: 10.1109/CEIDP.2002.1048793.

[3] M. Supova, G.S. Martynkova, K. Barabaszova, Effect of nanofillers dispersion in polymer matrices: a review, Sci. Adv. Mater. 3(1) (2011) 1-25, DOI: 10.1166/sam.2011.1136.

[4] D.L. Ma, T.A. Hugener, R.W. Siegel, A. Christerson, E. Martensson, C. Onneby, L.S. Schadler, Influence of nanoparticle surface modification on the electrical behaviour of polyethylene nanocomposites, Nanotechnology 16(6) (2005) 724-731, DOI: 10.1088/0957-4484/16/6/016.

[5] X.Y. Huang, Z.S. Ma, Y.Q. Wang, P.K. Jiang, Y. Yin, Z. Li, Polyethylene/aluminum nanocomposites: improvement of dielectric strength by nanoparticle surface modification, J. Appl. Polym. Sci. 113(6) (2009) 35773584, DOI: 10.1002/app.30313.

[6] Y. Li, T.M. Krentz, L. Wang, B.C. Benicewicz, L.S. Schadler, Ligand Engineering of Polymer Nanocomposites: From the Simple to the Complex, ACS Appl. Mater. Interfaces 6(9) (2014) 6005-6021, DOI: 10.1021/am405332a.

[7] X.Y. Huang, F. Liu, P.K. Jiang, Effect of nanoparticle surface treatment on morphology, electrical and water treeing behavior of LLDPE composites, IEEE Trns. Dielectr. Electr. Insul. 17(6) (2010) 1697-1704, DOI: 10.1109/TDEI.2010.5658219.

[8] M.L. Minus, H.G. Chae, S. Kumar, Polyethylene Crystallization Nucleated by Carbon Nanotubes under Shear, ACS Appl. Mater. Interfaces 4(1) (2012) 326-330, DOI: 10.1021/am2013757.

[9] I.L. Hosier, M. Praeger, A.F. Holt, A.S. Vaughan, S.G. Swingler, On the Effect of Functionalizer Chain Length and Water Content in Polyethylene/Silica Nanocomposites: Part I - Dielectric Properties and Breakdown Strength, IEEE Trns. Dielectr. Electr. Insul. 24(3) (2017) 1698-1707, DOI: 10.1109/tdei.2017.005788.

[10] I.L. Hosier, A.S. Vaughan, S.G. Swingler, On the effects of morphology and molecular composition on the electrical strength of polyethylene blends, J. Polym. Sci., Part B: Polym. Phys. 38(17) (2000) 2309-2322, DOI: 10.1002/1099-0488(20000901)38:17<2309::aid-polb110>3.3.co;2-z.

[11] C. Zou, J.C. Fothergill, S.W. Rowe, The effect of water absorption on the dielectric properties of epoxy nanocomposites, IEEE Trns. Dielectr. Electr. Insul. 15(1) (2008) 106-17, DOI: 10.1109/t-dei.2008.4446741.

[12] L. Hui, L.S. Schadler, J.K. Nelson, The influence of moisture on the electrical properties of crosslinked polyethylene/silica nanocomposites, IEEE Trns. Dielectr. Electr. Insul. 20(2) (2013) 641-653, DOI: 10.1109/TDEI.2013.6508768.

[13] I.L. Hosier, M. Praeger, A.F. Holt, A.S. Vaughan, S.G. Swingler, Effect of water absorption on dielectric properties of nano-silica/polyethylene composites, IEEE Conf. Electr. Insul. Dielectr. Phenom. (2014) 651-654, DOI: 10.1109/CEIDP.2014.6995816.

[14] D. Fabiani, G.C. Montanari, L. Testa, Effect of aspect ratio and water contamination on the electric properties of nanostructured insulating materials, IEEE Trns. Dielectr. Electr. Insul. 17(1) (2010) 221-230, DOI: 10.1109/TDEI.2010.5412021.

[15] I.L. Hosier, A.F. Holt, A.S. Vaughan, S.G. Swingler, On the effect of functionaliser chain length and water content in polyethylene/silica nanocomposites: Part II - Charge transport, IEEE Trns. Dielectr. Electr. Insul. 24(4) (2017) 2410-2420, DOI: 10.1109/TDEI.2017.005789. 
[16] S. Siddabattuni, T.P. Schuman, F. Dogan, Dielectric Properties of Polymer-Particle Nanocomposites Influenced by Electronic Nature of Filler Surfaces, ACS Appl. Mater. Interfaces 5(6) (2013) 1917-1927, DOI: 10.1021/am3030239.

[17] A. Mohanty, V.K. Srivastava, Dielectric breakdown performance of alumina/epoxy resin nanocomposites under high voltage application, Mater. Des. 47 (2013) 711-716, DOI: 10.1016/j.matdes.2012.12.052.

[18] J.K. Nelson, Nanodielectrics - the first decade and beyond, Int. Symp. Electr. Insul. Mater. (2014) 1-11, DOI: 10.1109/ISEIM.2014.6870707.

[19] M. Roy, J.K. Nelson, R.K. MacCrone, L.S. Schadler, C.W. Reed, R. Keefe, W. Zenger, Polymer nanocomposite dielectrics - The role of the interface, IEEE Trns. Dielectr. Electr. Insul. 12(4) (2005) 629-643, DOI: 10.1109/tdei.2005.1511089.

[20] P. Morshuis, Interfaces: To be avoided or to be treasured? What do we think we know?, IEEE Int. Conf. Solid Dielectr. (2013) 1-9, DOI: 10.1109/ICSD.2013.6619726.

[21] T.J. Lewis, Interfaces are the dominant feature of dielectrics at the nanometric level, IEEE Trns. Dielectr. Electr. Insul. 11(5) (2004) 739-753, DOI: 10.1109/tdei.2004.1349779.

[22] T.J. Lewis, Interfaces: nanometric dielectrics, J. Phys. D: Appl. Phys. 38(2) (2005) 202-212, DOI: 10.1088/0022-3727/38/2/004.

[23] G. Cao, Nanostructures \& Nanomaterials: Synthesis, Properties \& Applications, Imperial College Press, London, UK, 2004.

[24] T. Tanaka, M. Kozako, N. Fuse, Y. Ohki, Proposal of a multi-core model for polymer nanocomposite dielectrics, IEEE Trns. Dielectr. Electr. Insul. 12(4) (2005) 669-681, DOI: 10.1109/tdei.2005.1511092.

[25] R.B. Bogoslovov, C.M. Roland, A.R. Ellis, A.M. Randall, C.G. Robertson, Effect of silica nanoparticles on the local segmental dynamics in poly(vinyl acetate), Macromolecules 41(4) (2008) 1289-1296, DOI: 10.1021/ma702372a.

[26] C.G. Robertson, C.J. Lin, M. Rackaitis, C.M. Roland, Influence of particle size and polymer-filler coupling on viscoelastic glass transition of particle-reinforced polymers, Macromolecules 41(7) (2008) 2727-2731, DOI: 10.1021/ma7022364.

[27] S.E. Harton, S.K. Kumar, H.C. Yang, T. Koga, K. Hicks, E. Lee, J. Mijovic, M. Liu, R.S. Vallery, D.W. Gidley, Immobilized polymer layers on spherical nanoparticles, Macromolecules 43(7) (2010) 3415-3421, DOI: 10.1021/ma902484d.

[28] A. Sargsyan, A. Tonoyan, S. Davtyan, C. Schick, The amount of immobilized polymer in PMMA SiO2 nanocomposites determined from calorimetric data, Eur. Polym. J. 43(8) (2007) 3113-3127, DOI: 10.1016/j.eurpolymj.2007.05.011.

[29] A.P. Holt, P.J. Griffin, V. Bocharova, A.L. Agapov, A.E. Imel, M.D. Dadmun, J.R. Sangoro, A.P. Sokolov, Dynamics at the polymer/nanoparticle interface in poly(2-vinylpyridine)/silica nanocomposites, Macromolecules 47(5) (2014) 1837-1843, DOI: 10.1021/ma5000317.

[30] V. Bershtein, V. Gun'ko, L. Egorova, N. Guzenko, E. Pakhlov, V. Ryzhov, V. Zarko, Well-defined silica corepoly(vinyl pyrrolidone) shell nanoparticles: Interactions and multi-modal glass transition dynamics at interfaces, Polymer 50(3) (2009) 860-871, DOI: 10.1016/j.polymer.2008.12.024.

[31] F.N. Alhabill, R. Ayoob, T. Andritsch, A.S. Vaughan, Effect of resin/hardener stoichiometry on electrical behavior of epoxy networks, IEEE Trns. Dielectr. Electr. Insul. 24(6) (2017) 3739-3749, DOI: 10.1109/TDEI.2017.006828.

[32] J.K. Nelson, Y.H. Huang, T.M. Krentz, L.S. Schadler, J. Dryzek, B.C. Benicewicz, M. Bell, Free Volume in Nanodielectrics, IEEE International Conference on the Properties and Applications of Dielectric Materials (2015) 40-43, DOI: 10.1109/ICPADM.2015.7295203.

[33] K.J. Klabunde, Nanoscale materials in chemistry, John Wiley \& Sons, Inc.2001. 
[34] J.A. Anta, G. Marcelli, M. Meunier, N. Quirke, Models of electron trapping and transport in polyethylene: Current-voltage characteristics, J. Appl. Phys. 92(2) (2002) 1002-1008, DOI: 10.1063/1.1489714.

[35] L.A. Dissado, J.C. Fothergill, Electrical degradation and breakdown in polymers, Institution of Engineering and Technology1992.

[36] R.C. Smith, C. Liang, M. Landry, J.K. Nelson, L.S. Schadler, The mechanisms leading to the useful electrical properties of polymer nanodielectrics, IEEE Trns. Dielectr. Electr. Insul. 15(1) (2008) 187-196, DOI: 10.1109/tdei.2008.4446750.

[37] S. Le Roy, G. Teyssedre, C. Laurent, G.C. Montanari, F. Palmieri, Description of charge transport in polyethylene using a fluid model with a constant mobility: fitting model and experiments, J. Phys. D: Appl. Phys. 39(7) (2006) 1427-1436, DOI: 10.1088/0022-3727/39/7/014.

[38] M. Roy, J.K. Nelson, R.K. MacCrone, L.S. Schadler, Candidate mechanisms controlling the electrical characteristics of silica/XLPE nanodielectrics, J. Mater. Sci. 42(11) (2007) 3789-3799, DOI: 10.1007/s10853-0060413-0.

[39] M. Reading, A.S. Vaughan, P.L. Lewin, An investigation into improving the breakdown strength and thermal conduction of an epoxy system using boron nitride, IEEE Conf. Electr. Insul. Dielectr. Phenom. (2011) 636-9, DOI: 10.1109/ceidp.2011.6232737.

[40] Z. Xu, M. Reading, A.S. Vaughan, P.L. Lewin, Space charge distribution in filled epoxy composites containing micro and nano fillers, IEEE International Symposium on Electrets (2011) 117-118, DOI: 10.1109/ISE.2011.6085010.

[41] K.Y. Lau, A.S. Vaughan, G. Chen, I.L. Hosier, A.F. Holt, K.Y. Ching, On the space charge and DC breakdown behavior of polyethylene/silica nanocomposites, IEEE Trns. Dielectr. Electr. Insul. 21(1) (2014) 340-351, DOI: 10.1109/tdei.2013.004043.

[42] M. Praeger, I.L. Hosier, A.S. Vaughan, S.G. Swingler, The effects of surface hydroxyl groups in polyethylenesilica nanocomposites, IEEE Electr. Insul. Conf. (2015) 201-204, DOI: 10.1109/ICACACT.2014.7223514.

[43] E. Kubyshkina, B.L.G. Jonsson, M. Unge, Electronic properties of magnesium oxide - polyethylene interface, IEEE International Conference on Dielectrics 2 (2016) 788-791, DOI: 10.1109/ICD.2016.7547734.

[44] Y.L. Li, Y. Liang, F. Zheng, K. Xiao, Z.Q. Hu, T. Shun, Fourier transformation infrared investigation of surface oxidation of ultrafine Si3N4 powders, J. Mater. Sci. Lett. 14(10) (1995) 713-715.

[45] Y.L. Tai, J.S. Qian, J.B. Miao, R. Xia, Y.C. Zhang, Z.G. Yang, Preparation and characterization of Si3N4/SBR nanocomposites with high performance, Mater. Des. 34 (2012) 522-527, DOI: 10.1016/j.matdes.2011.05.002.

[46] F.N. Alhabill, T. Andritsch, A.S. Vaughan, The effect of resin/hardener stoichiometry on the electrical properties of silicon nitride/epoxy nanocomposites IEEE International Conference on Dielectrics (2016), DOI: 10.1109/ICD.2016.7547553.

[47] F.N. Alhabill, R. Ayoob, T. Andritsch, A.S. Vaughan, Influence of filler/matrix interactions on resin/hardener stoichiometry, molecular dynamics, and particle dispersion of silicon nitride/epoxy nanocomposites, J. Mater. Sci. 53(6) (2018) 4144-4158, DOI: 10.1007/s10853-017-1831-x.

[48] B. Fubini, M. Volante, V. Bolis, E. Giamello, Reactivity towards water of silicon nitride: energy of interaction and hydration dehydration mechanism J. Mater. Sci. 24(2) (1989) 549-556, DOI: 10.1007/bf01107440.

[49] W. Liwu, W. Sigmund, F. Aldinger, Systematic approach for dispersion of silicon nitride powder in organic media. I. Surface chemistry of the powder, J. Am. Ceram. Soc. 83(4) (2000) 691-6, DOI: 10.1111/j.11512916.2000.tb01261.x.

[50] G.S. Wu, L.C. Ma, L. Liu, Y.W. Wang, F. Xie, Z.X. Zhong, M. Zhao, B. Jiang, Y.D. Huang, Interface enhancement of carbon fiber reinforced methylphenylsilicone resin composites modified with silanized carbon nanotubes, Mater. Des. 89 (2016) 1343-1349, DOI: 10.1016/j.matdes.2015.10.016.

[51] Y.H. Tang, L. Ye, S.Q. Deng, C. Yang, W.Z. Yuan, Influences of processing methods and chemical treatments on fracture toughness of halloysite-epoxy composites, Mater. Des. 42 (2012) 471-477, DOI: 10.1016/j.matdes.2012.06.036. 
[52] C.P. Tripp, M.L. Hair, Reaction of methylsilanols with hydrated silica surfaces - the hydrolysis of trichloromethylsilanes, dichloromethylsilanes, and monochloromethylsilanes and the effects of curing, Langmuir 11(1) (1995) 149-155, DOI: 10.1021/la00001a027.

[53] M.M. Sung, G.J. Kluth, R. Maboudian, Formation of alkylsiloxane self-assembled monolayers on Si3N4, J. Vac. Sci. Technol., A 17(2) (1999) 540-544, DOI: 10.1116/1.581616.

[54] I.L. Hosier, M. Praeger, A.S. Vaughan, S.G. Swingler, Electrical properties of polymer nano-composites based on oxide and nitride fillers, IEEE Electr. Insul. Conf. (2015) 438-441, DOI: 10.1109/ICACACT.2014.7223515.

[55] F.N. Alhabill, T. Andritsch, A.S. Vaughan, Effect of the processing method on the electrical behavior of silicon nitride/epoxy nanocomposites, IEEE Conf. Electr. Insul. Dielectr. Phenom. (2015) 511-514, DOI: 10.1109/CEIDP.2015.7352133.

[56] X.J. Chen, J.G. Jiang, F. Yan, S.C. Tian, K.M. Li, A novel low temperature vapor phase hydrolysis method for the production of nano-structured silica materials using silicon tetrachloride, RSC Adv. 4(17) (2014) 8703-8710, DOI: $10.1039 / \mathrm{c} 3 \mathrm{ra} 47018 \mathrm{k}$.

[57] J. Szepvolgyi, I. Mohai, J. Gubicza, Atmospheric ageing of nanosized silicon nitride powders, J. Mater. Chem. 11(3) (2001) 859-863, DOI: 10.1039/B008429H.

[58] L.G. Wade, Organic Chemistry, 8 ed., Pearson Education2013.

[59] A.M. Pourrahimi, L.K.H. Pallon, D. Liu, T.A. Hoang, S. Gubanski, M.S. Hedenqvist, R.T. Olsson, U.W. Gedde, Polyethylene Nanocomposites for the Next Generation of Ultralow-Transmission-Loss HVDC Cables: Insulation Containing Moisture-Resistant MgO Nanoparticles, ACS Appl. Mater. Interfaces 8(23) (2016) 14824-14835, DOI: 10.1021/acsami.6b04188.

[60] D.K. Shukla, V. Parameswaran, Epoxy composites with $200 \mathrm{~nm}$ thick alumina platelets as reinforcements, J. Mater. Sci. 42(15) (2007) 5964-5972, DOI: 10.1007/s10853-006-1110-8.

[61] M.M. Shokrieh, A.R. Kefayati, M. Chitsazzadeh, Fabrication and mechanical properties of clay/epoxy nanocomposite and its polymer concrete, Mater. Des. 40 (2012) 443-452, DOI: 10.1016/j.matdes.2012.03.008.

[62] F.W. Starr, J.F. Douglas, S.C. Glotzer, Origin of particle clustering in a simulated polymer nanocomposite and its impact on rheology, J. Chem. Phys. 119(3) (2003) 1777-1788, DOI: 10.1063/1.1580099.

[63] Y.F. Luo, Y. Zhao, J.Z. Cai, Y.X. Duan, S.Y. Du, Effect of amino-functionalization on the interfacial adhesion of multi-walled carbon nanotubes/epoxy nanocomposites, Mater. Des. 33 (2012) 405-412, DOI: 10.1016/j.matdes.2011.04.033.

[64] Q.J. Zhang, J.Q. Wu, L. Gao, T. Liu, W.H. Zhong, G. Sui, G.D. Zheng, W. Fang, X.P. Yang, Dispersion stability of functionalized MWCNT in the epoxy-amine system and its effects on mechanical and interfacial properties of carbon fiber composites, Mater. Des. 94 (2016) 392-402, DOI: 10.1016/j.matdes.2016.01.062.

[65] C.L. Soles, A.F. Yee, A discussion of the molecular mechanisms of moisture transport in epoxy resins, J. Polym. Sci., Part B: Polym. Phys. 38(5) (2000) 792-802, DOI: 10.1002/(sici)1099-0488(20000301)38:5<792::aidpolb16>3.0.co;2-h.

[66] C. Jordan, J. Galy, J.P. Pascault, Measurement of the extent of reaction of an epoxy-cycloaliphatic amine system and influence of the extent of reaction on its dynamic and static mechanical-properties, J. Appl. Polym. Sci. 46(5) (1992) 859-871, DOI: 10.1002/app.1992.070460513.

[67] S. Cukierman, Et tu, Grotthuss! and other unfinished stories, Biochimica Et Biophysica Acta-Bioenergetics 1757(8) (2006) 876-885, DOI: 10.1016/j.bbabio.2005.12.001.

[68] T. Sameshima, K. Sakamoto, Y. Tsunoda, T. Saitoh, Improvement of SiO2 properties and silicon surface passivation by heat treatment with high-pressure H2O vapor, Jpn. J. Appl. Phys., Part 2 37(12A) (1998) L1452L1454, DOI: 10.1143/jjap.37.11452.

[69] H.R. Philipp, Optical properties of silicon nitride, J. Electrochem. Soc. 120(2) (1973) 295-300, DOI: 10.1149/1.2403440. 
